\title{
Overview Of EDFA for the Efficient Performance Analysis
}

\author{
Anuja Dhokar ${ }^{1}$, S.D.Deshmukh ${ }^{2}$ \\ ${ }^{\text {I}}$ (M.E Student, Department of Electronics \& Telecommunication, Rajiv Gandhi Institute of Tech., \\ Mumbai University, India) \\ ${ }_{2}^{2}$ (Asst. Professor, Department of Electronics \& Telecommunication, Rajiv Gandhi Institute of Tech., \\ Mumbai University, India)
}

\begin{abstract}
Fiber loss is a fundamental limitation in realizing long haul point-to-point fiber optical communication links and optical networks. One of the advanced technologies achieved in recent years is the advent of Erbium doped fiber amplifier (EDFA) it is an essential element in DWDM networks. This all-optical amplifier enables instantaneous amplification of multiple wavelengths in regard of electronic regeneration; direct optical amplification using erbium-doped fiber amplifiers offers many advantages for long haul repeater transmission. First, the repeater can be quite simply configured regardless of the line signal bit rate. This feature becomes more significant as the line signal bit rate exceeding $1 \mathrm{~Tb} / \mathrm{s}$ at which speed electronic regeneration requires high-speed electronic circuits, thereby resulting in increase in hardware cost and power consumption.In this paper EDFA has been studied to characterize Gain, Noise Figure of a forward pumped EDFA operating in C band $(1525-1565 \mathrm{~nm})$ as functions of $\mathrm{Er}+3$ fiber length, injected pump power, signal input power and $\mathrm{Er}+3$ doping density.
\end{abstract}

Keywords: - Wavelength Division Multiplexing(WDM), Dence Wavelength Division Multiplexing(DWDM), Gain, Noise Figure (NF)

\section{INTRODUCTION}

The erbium-doped fiber amplifier EDFA invented in 1986 and has made tremendous progress. It replaced the rather involved process of the fiber optic repeater station. It created a revolution in long distance optical communication systems. Simplicity and reliability of the repeater compartment are especially important when the optical fiber cable is used as a submarine cable [1].Erbium Doped Fiber Amplifiers (EDFA) made by doping the silica fiber with erbium ions can operate in a broad range within the $1550 \mathrm{~nm}$ window at which the attenuation of silica fiber is minimum and therefore it is ideal for the optical fiber communication systems operating at this wavelength range. According to the research performed in recent years, it is known that the pumping of erbium doped fiber at $980 \mathrm{~nm}$ or $1480 \mathrm{~nm}$ is the most efficient way [2].High gain (30 50dB), large bandwidth $(>90 \mathrm{~nm})$, high output power $(10 \sim 20 \mathrm{dBm}$ )and low noise figure $(\mathrm{NF}=3 \sim 5 \mathrm{~dB})$ can be obtained using an erbium doped fiber amplifier optimized for $1550 \mathrm{~nm}$ range [3].

\section{BACKGROUND}

The amplifier is modeled as a three-level system [4], having Three populations of erbium atoms are of interest here: 1) the ground state with population density $\mathrm{n} 1$;2) metastable level with population density $\mathrm{n} 2$; and 3) pump level with population density $\mathrm{n} 3$. In practice, transitions from 3 to 2 are much more likely than transitions back to the ground state ( 3 to 1 ) or the rate of spontaneous emission from state 2 . Under these assumptions, $\mathrm{n} 3 \approx 0$. The rate equations describing the effects of the pump $\left(\mathrm{P}_{\mathrm{P}}\right)$,signal $\left(\mathrm{P}_{\mathrm{S}}\right)$, and ASE $\left(\mathrm{P}_{\mathrm{ASE}}\right)$ power reduces to the following for $\mathrm{n} 2$ :

$$
\frac{d n_{2}(z, t)}{d t}=\frac{\tau_{g} \sigma_{12}}{h v_{2} A}\left(P_{g}+P^{+}{ }_{A S E}+P^{-}{ }_{A S E}\right) n_{2}-\frac{n_{2}}{\tau}-\frac{\tau_{p} \sigma_{13}}{h v_{p} A}\left(P_{p}^{+}+P_{p}^{-}\right) n_{2}
$$

A is the effective cross-sectional area of the core. The $\sigma_{g}^{*}$ are known absorption and emission crosssection data for the erbium fiber. The $\boldsymbol{\tau}_{\mathrm{s}}$ are the mode confinement factors for the pump and signal waves. The superscript + designates pump ASE co propagating with the signal, and - when they counter propagate to the signal. In modern fibers we can neglect scattering and other losses, so the convective equations describing the spatial development of the pump, signal and ASE in the fiber are[4]:

$\frac{d P_{s}(z, t)}{d z}=P_{p} \tau_{s}\left(\sigma_{12} n_{2}-\sigma_{21} n_{1}\right)$ 


$$
\begin{aligned}
& \frac{d P_{s}(z, t)}{d z}=P_{p} \tau_{s}\left(\sigma_{12} n_{2}-\sigma_{21} n_{1}\right) \\
& \frac{d P_{A S E}^{ \pm}(z, t)}{d z}= \pm P_{A S E}^{ \pm} \tau_{s}\left(\sigma_{21} n_{2}-\sigma_{12} n_{1}\right) \pm 2 \sigma_{21} \tau_{s} h v_{s} \Delta v n_{2}
\end{aligned}
$$

Equations (1) and (2) are the basic equations describing an EDFA.

\section{DYNAMICAL MODEL}

For dynamical modeling, first (2) is substituted into (1) and then integrated over $\mathrm{Z}$ to remove the length dependence of erbium-doped fiber [5]. This results in a dynamic equation for the excited state population $N_{2}$, which in turn determines the dynamics of the output pump and communication signals.

$$
\frac{d}{d t}\left(n_{2} A\right)=-\frac{d P_{s}}{d z}-\frac{d P_{p}^{+}}{d z}-\frac{n_{2} A}{\tau}-\frac{d P_{A S E}^{+}}{d z}+2 \sigma_{21} \tau_{s} h v_{s} n_{2} \Delta v
$$

In equation (3) we include only co propagating pump and ASE. Integrating (3) over Z, we have

$$
\frac{d}{d t} N_{2}=P_{s}(0, t)-P_{s}(0, t)+P_{p}^{+}(0, t)-P_{p}^{+}(L, t)-\frac{N_{2}}{\tau}+P_{A S E}^{+}(0, t)-P_{A S E}^{+}(L, t)
$$

Where $N 2$ is the number of erbium ions in the excited state. $P \mathrm{~S}(0, \mathrm{t})$ and $P \mathrm{p}(0, \mathrm{t})$ represent the time-dependent input powers for the pump and signal. The output powers $P \mathrm{~S}(\mathrm{~L}, \mathrm{t})$ and $P \mathrm{p}(\mathrm{L}, \mathrm{t})$ are explicit functions of $N 2$.

$$
\begin{aligned}
\frac{d}{d t} N_{2}=P_{s}(0, t) & {\left[1-\exp \left(B_{S} N_{2}-C_{S}\right)\right]+P_{P}^{+}(0, t)[1} \\
& \left.-\exp \left(B_{P} N_{2}-C_{P}\right)\right]-\frac{N_{2}}{\tau}-2 h v_{s} n_{S p} \Delta v(G-1)+2 h v_{s} \frac{\beta_{S}}{\rho A} \Delta v N_{2}
\end{aligned}
$$

This is the key equation for considering dynamic gain effects in the EDFA including ASE. In equation (5) $\mathrm{G}$ is the gain, $\beta s$ is the emission per unit length, $\Delta v$ and $v$ refer to the wavelength deviation of the ASE power around $\lambda, h$ is Planks constant, and $n s p$ is the population-inversion factor which is dimensionless [6]. In an EDFA, complete inversion can only be obtained when being pumped at $980 \mathrm{~nm}$; at $980 \mathrm{~nm} \beta P=0$ and therefore $n s p=1$. So a pump wavelength of $980 \mathrm{~nm}$ is assumed. The $\mathrm{B}$ and $\mathrm{C}$ are given in terms of the confinement factors $\boldsymbol{\tau}_{s}$ and $\boldsymbol{\tau}_{p}$, the absorption and emission cross sections $\left(\sigma_{12}, \sigma_{21}\right.$, and $\left.\sigma_{13}\right)$, the density of erbium atoms $\rho$, the length $\mathrm{L}$, and the effective cross sectional area $\mathrm{A}$ of the erbium-doped fiber. $\tau$ is the rate of spontaneous emission, and $r$ is the effective radius of the fiber core.

\section{GAIN MODULATION}

To model the impact of modulation, we add perturbations to the pump and signal transition rates. Over modulation is introduced as a sinusoidal time variation of the pump or signal power [3], described by

$P_{p, S}(0, t)=P_{P, S}^{0}(0)\left(1+m_{P, S} \cos \omega t\right)$

Here, $P^{0}(0)$ is the mean power (pump or signal) at the input ( $\left.\mathrm{z}=0\right)$ and $m p, s$ is the input modulation index. Note the over modulation frequency is assumed small $(\sim \mathrm{kHz})$ compared with the communications signal data rate $(\sim \mathrm{Gb} / \mathrm{s})$ and can be considered simply as an analog modulation imposed on the mean signal power power. The EDFA over modulation behavior is then obtained by solving (5) with time-dependent inputs of the form of (6). As a first step, we expand $N^{2}$ (t) about its mean (unmodulated) steady-state solution $N^{0}{ }_{2}$

$N_{2}(t)=N_{2}^{0}(1+\delta \cos (\omega t+\varphi))$

\section{Pump and signal Modulation}

Using the method described by Novak and Mosle, the equations for the amplitude and phase of the pump-tosignal transfer function are given by 


$$
\begin{aligned}
& m_{p}^{\prime}=\frac{m_{p} B_{S}\left[P_{p}^{0}(0)-P_{p}^{0}(L)\right.}{\sqrt{\omega^{2}+\omega_{e f f}^{2}}} \\
& \tan \theta_{p}=-\frac{\omega}{\omega_{\text {eff }}} \\
& \omega_{\text {eff }}=P_{S}^{0}(L)+\frac{1}{\tau}+2 n_{S p} \Delta v G B_{S}-2 \Delta v \frac{\beta_{S}}{\rho A}
\end{aligned}
$$

And for the amplitude and phase of the signal-to-signal transfer function are given by

$$
\begin{gathered}
m_{S}^{v}=\frac{m_{S} \sqrt{\omega^{2}+\left(\omega_{\text {eff }}+K\right)^{2}}}{\sqrt{\omega^{2}+\omega_{\text {eff }}^{2}}} \\
K=B_{S}\left[P_{S}^{0}(0)-P_{S}^{0}(L) \quad\right. \text { and }
\end{gathered}
$$

$\tan \theta_{S}=-\frac{\omega}{\omega_{\text {eff }}+\frac{\omega^{2}+\omega_{\text {eff }}^{2}}{K}}$

These expressions describe the output signal modulation index amplitude and phase, assuming small sinusoidal steady-state oscillations of the mean pump or input signal power .

\section{THE STRUCTURE OF EDFA AND IT'S PUMPING REQUIREMENTS}

The structure of a typical EDFA is shown in figure1. EDFAs consist of optical couplers to combine pump and signal lights injected to active fiber. The gain characteristics of EDFAs can be pumped at $980 \mathrm{~nm}$ or $1480 \mathrm{~nm}$, and with different configurations: backward, forward or bidirectional. In forward pumping, both of the signal and pump lights propagate in the same direction through the fiber whereas in the backward pumping they propagate in the opposite direction.

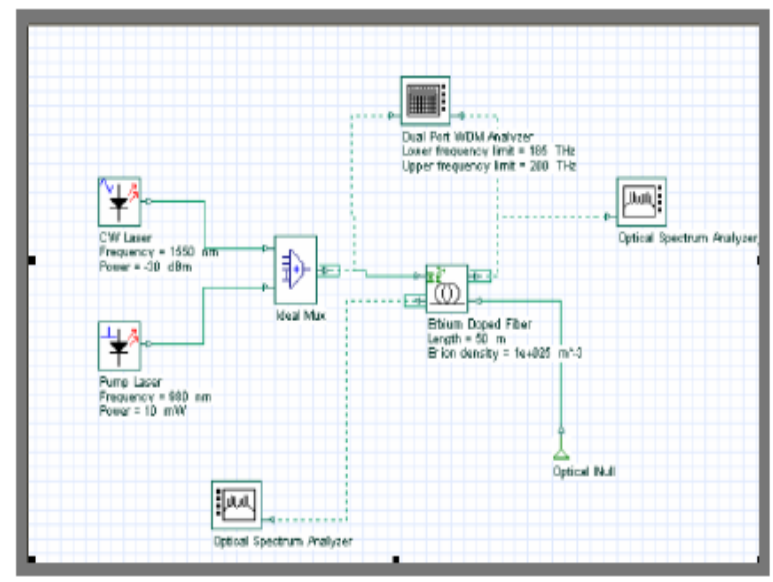

figure1. Forward pumped EDFA structure [5]

\section{Gain Characteristics}

The variation of gain with fiber length is shown in figure (2.a) for different pump powers having a constant signal input power and erbium doping density. The gain varies along the fiber length because of pump power variations. For a given amplifier length, the amplifier gain initially increases exponentially with the pump power and then goes to saturation after a certain level of pump power. For a given pump power the amplifier gain increases up to a certain length of fiber, and then begins to decrease after a maximum point. The physical considerations for the decrease in gain is insufficient population inversion due to excessive pump depletion and 
getting higher losses than the provided gain at the signal wavelength due to high total loss of Erbium doped fiber (fiber background loss+ Er absorption loss).

Figure (2.b) shows the variation of gain with pump Power for different fiber lengths $(10,30,50) \mathrm{m}$, pumping power is swept from (0 to 100$) \mathrm{mw}$, and erbium ion density $=1 \times 1024 \mathrm{~m}-3$ (100 ppm-wt).

It is seen that the gain of EDFA sharply increases with the increasing pump power. After a certain level of gain, the increase in gain becomes smaller when the population inversion is provided for all the erbium ions in the fiber and therefore amplifier goes to saturation, in addition, a higher gain can be obtained if a longer erbium doped fiber is used with sufficient pumping.

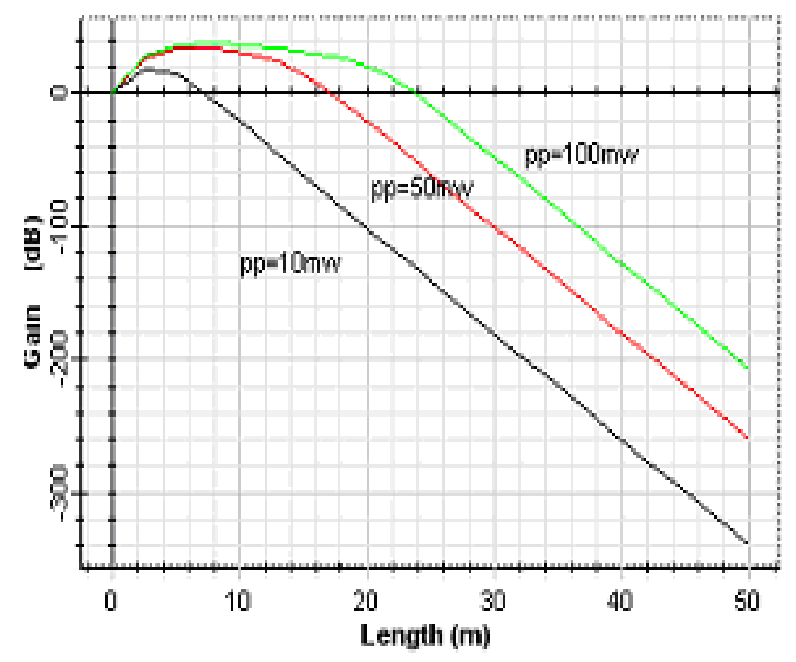

figure 2.the variation of gain with a) fiber length and

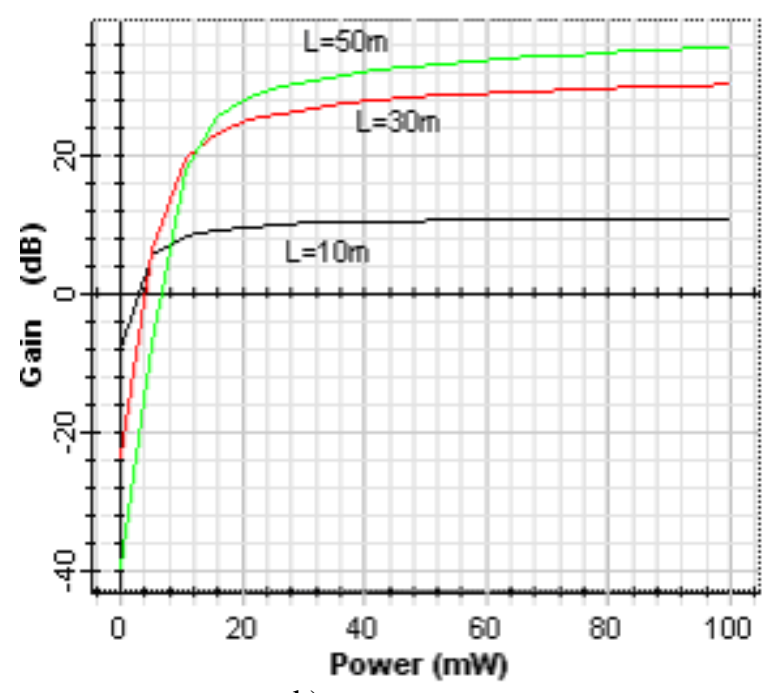

b) pump power

Figure (3.a) shows how the gain varies as a function of signal input power for different pumping powers $(10,50 \mathrm{and} 100 \mathrm{mw})$, constant fiber length $(50 \mathrm{~m})$ and erbium doping density $(100 \mathrm{ppm}-\mathrm{wt})$, signals power was swept from(- $-40 \mathrm{dBm}$ to $5 \mathrm{dBm})$. It is seen that EDFA gain decreases with the increasing signal input power. When signal power less than $-30 \mathrm{dBm}$ the amplifier works in small - signal regime where the signal gain is independent of the input signal power indeed the signal power is very weak and the amplifier works in unsaturated gain regime. When the amplifier reaches the saturation the maximum gain dropped by $3 \mathrm{~dB}$ below its unsaturated value Gmax. The physical meaning of this is the easier saturation of the EDFA at higher signal powers for a constant pump power. 


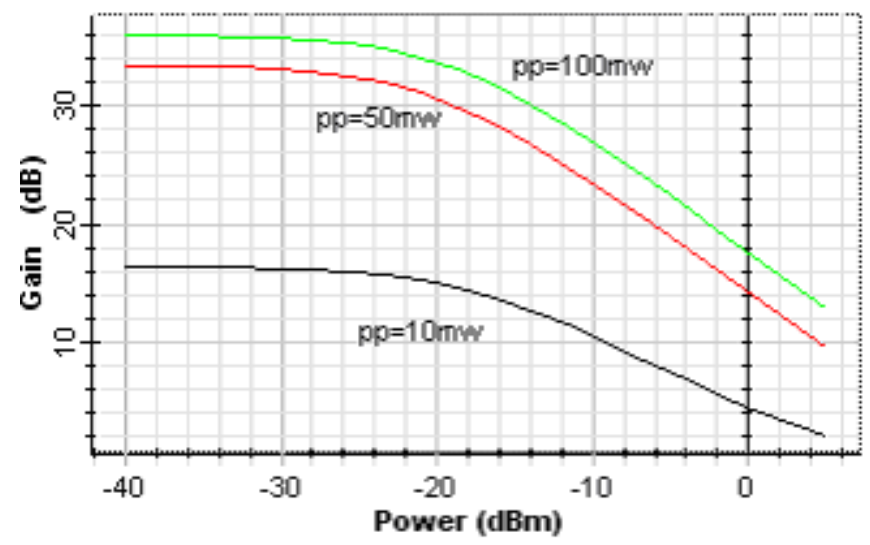

figure 3.The variation of gain with a)signal input power

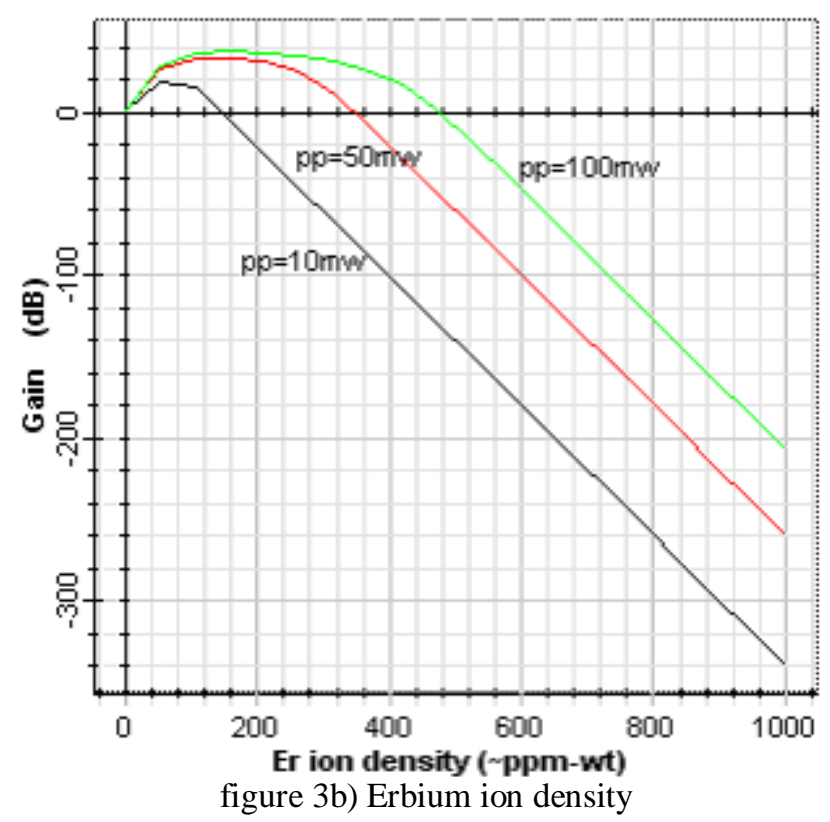

The gain variation as a function of erbium doping density is shown in figure $3 . \mathrm{b}$ for a $50 \mathrm{~m}$ long fiber and a constant signal input power for three different pump powers $(10 \mathrm{mw}, 50 \mathrm{mw}$ and $100 \mathrm{mw})$. It can be seen that for sufficiently large pump power, the gain linearly increases with increasing erbium ion density and remains constant after a certain level then decreases. Once the amplifier reaches the population inversion, the variation in maximum gain is small despite a high increase in pump power. In the trace obtained for $10 \mathrm{mw}$ pump power the gain reduces sharply in highly doped fiber due to insufficient pump.

\section{Noise Figure (NF) Characteristics}

The variation of noise figure as a function of fiber length is shown in figure (4.a) for different pumping powers at a constant signal input power and erbium ion density(1000ppm-wt). For a pump power of $10 \mathrm{mw}$ the increase in noise figure from $8 \mathrm{~m}$ can b e clearly noticed. The reason for this increase is the decreasing gain with sharp pump depletion. 


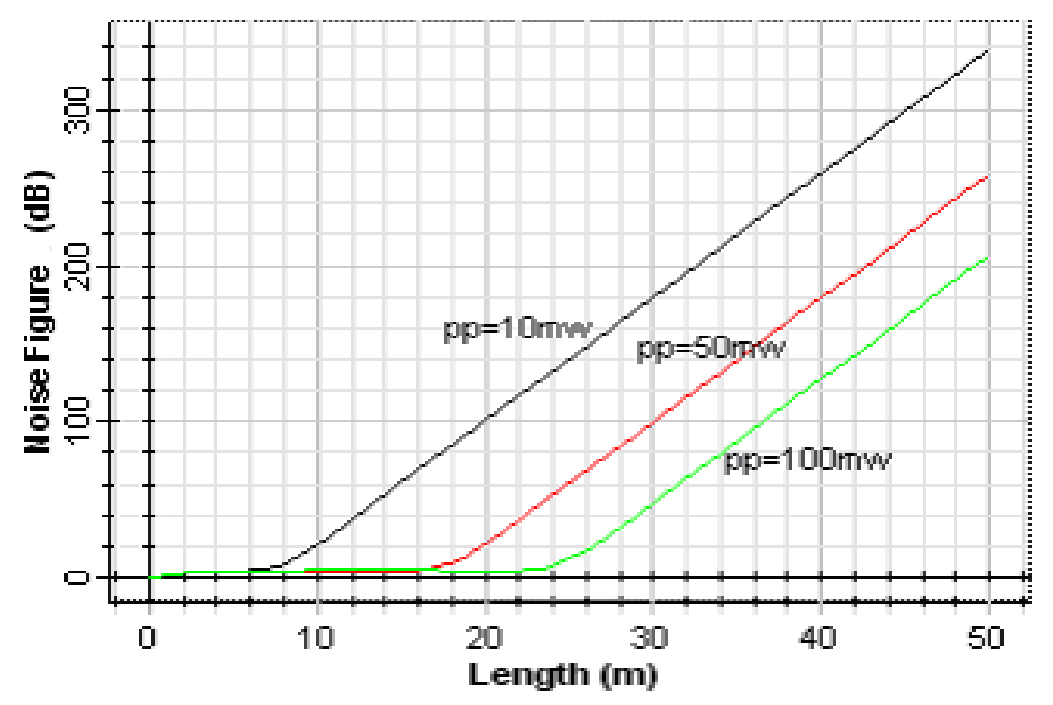

figure 4. The variation of noise figure with a) fiber length

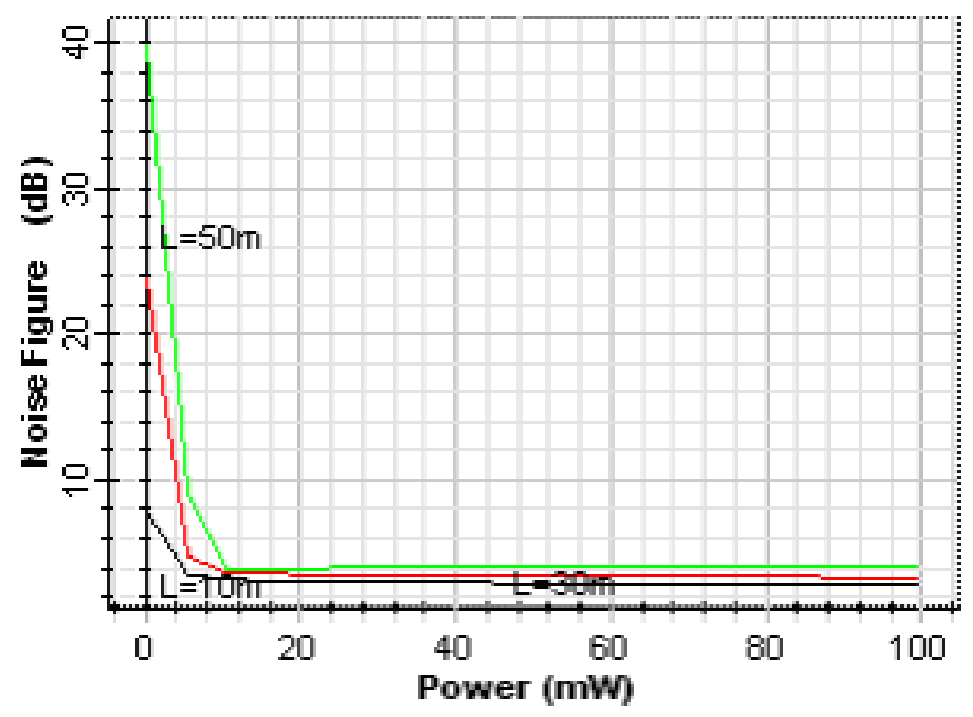

b) pump power

Figure (4.b) shows the noise figure variations as function of pump power for different fiber lengths $(10,30$ and $50 \mathrm{~m})$ at a constant signal input power and doping density as mentioned before. In an amplifier having those parameters, it can be seen that the noise figure decreases with increasing the pump power, at low pump power the noise figure is large for longer fiber length than shorter fiber, this is due to the insufficient pump power needed to obtain high gain in an active fiber, because high gain in an active fiber with the total population inversion causes the spontaneous emission to stay in low levels. The noise figure of an EDFA varies linearly with Amplified Spontaneous Emission (ASE) power and inversely with the amplifier gain; therefore, the NF of an EDFA can be reduced to a minimum level by increasing the gain.

In figure 5.a the variation of noise figure is given as a function of signal input power for a constant fiber length and erbium ion density. In this simulation a 50m long EDFA with an erbium ion density of 100ppm was used. The graph shows that the NF of an EDFA increases with increasing signal input power. The variation of noise figure with $\mathrm{Er}+3$ ion density is given in figure (5.b) for a constant fiber length and signal input power. Erbium ion density was taken from (1 to $1000 \mathrm{ppm}$-wt). It is seen that the noise figure remains constant nearly $(3 \mathrm{~dB})$ in a certain value of erbium ion density even if the pumping power is increased. Beyond 120ppm and for a $10 \mathrm{mw}$ pumping power, insufficient pumping occurs and the noise figure sharply increases and for $50 \mathrm{mw}$ pumping power beyond $315 \mathrm{ppm}$ the noise figure sharply increases due to insufficient population inversion. 


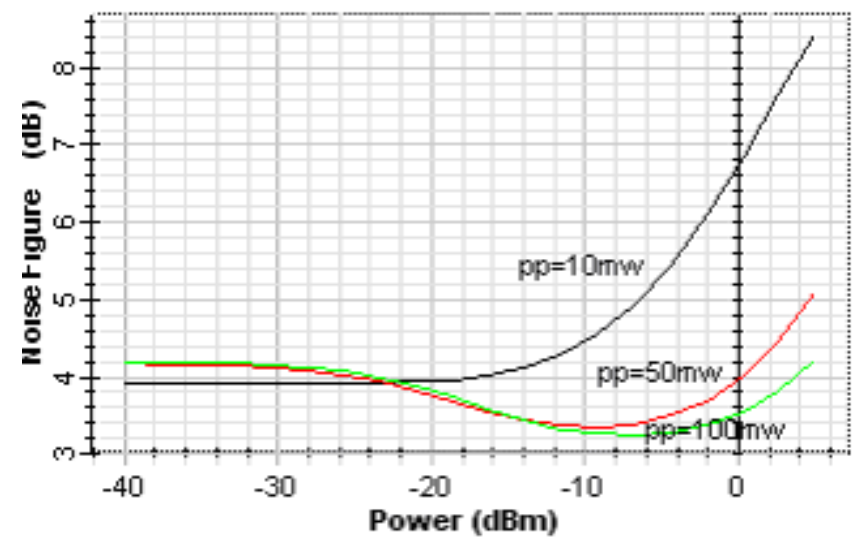

figure5. The variation of noise figure with a) signal input power

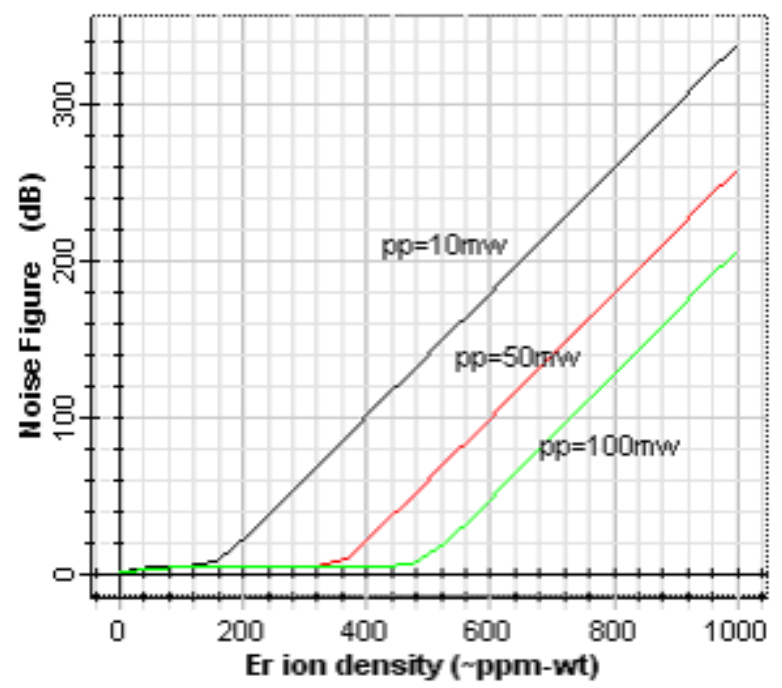

figure 5 b) Erbium ion density

\section{CONCLUSION}

In this study, the performance characteristic of EDFA operating in $C$ band and pumped at $980 \mathrm{~nm}$ simulated: Gain and noise figure variations were obtained as functions of fiber length, pump power, signal input power and erbium doping density in high bit rate 10Gbps. The gain varies along the fiber length because of pump power variations. When the EDFA is supplied with sufficient pump power, it was shown that EDFA could be operated in saturation regimes leading to maximum gain and minimum NF. It was seen that the variation of gain and noise figure as functions of fiber length, pump power, signal input power and erbium doping density do not change when bit rate is increased from $(2.5$ to $10 \mathrm{Gbps})$.

\section{ACKNOWLEDGEMENT}

The author would like to thank the following for valuable discussions related to this work: Prof. S.D.Deshmukh, Prof. Anamika Singh and Prof. S.D.Patil.

\section{REFERENCES}

[1] Keigo Iizuka, Erbium Doped Fiber Amplifier, advanced Labs for especial Topic in Photonics, Faculty Of Applied Science and Engineering University Of Toronto Canada, April 2003.

[2] Emmanuel Desurvire "Erbium Doped Fiber Amplifiers" Principles And Applications, Republished in New York by Colombia University 1994.

[3] http://en.wikipedia.org/wiki/optical_fiber.

[4] C.Giles, and Emmanuel Desurvire "Propagation of Signal and Noise in Concatenated Erbium-Doped Fiber Optical Amplifiers" J. Lightwave Te-chnol ., vol. 9. NO. 2. FEBRUARY 1991

[5] Optiwave Corporation "OptiAmplifier5.0 Component Library"2006. 
[6] B.S.Wang, G.Puc,M.Anderejco"Novel Erbium Doped Fiber for High Power Applications"OFS Labrotory,25 Schoolhouse Road, Somerset, NJ 08873,USA, Published in SPIE APOC2004.

[7] M. Murakami,T. Imai, and M. Aoyama, "A remote supervisory system based on subcarrier overmodulation for submarine optical amplifier systems," J. Lightwave Technol., vol. 14, pp. 671-677, May 1996.

[8] Agrawal, G. P, Fiber-Optic Communication Systems 3nd Ed, John Wiley and Sons,Sydney, 2002 ,pp. 250-261.

[9] S. Novak and A. Moesle, "Analytic model for gain modulation in EDFAs , "J. Lightwave Technol., vol. 20, pp. 975-985, June 2002.

[10] C.Giles, and Emmanuel Desurvire "Propagation of Signal and Noise in Concatenated Erbium-Doped Fiber Optical Amplifiers” J. Lightwave Te-chnol ., vol. 9. NO. 2. FEBRUARY 1991

[11] A P.C. Becker, N.A. Olsson, and J.R. Simpson, Erbium-Doped Fiber Ampl- ifiers Fundamentals and Technology. San Diego: Academic Press, 1999.

[12] J. Freeman and J. Conradi, "Gain modulation response of erbium-doped fiber amplifiers," IEEE Photon. Technol. Lett., vol. 5,pp. 224-226, Feb. 1993. 